\title{
Stereotactic body radiotherapy for lung metastases from colorectal cancer: a single institution experience
}

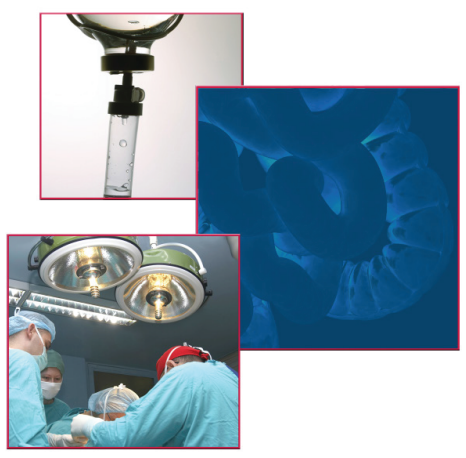

\author{
Rodney E Wegner*,1, Nissar Ahmed ${ }^{1}$, Shaakir Hasan ${ }^{1}$, James McCormick², Alexander \\ $\checkmark$ Kirichenko ${ }^{1}$ \& Athanasios Colonias $^{1}$ \\ ${ }^{1}$ Division of Radiation Oncology, Allegheny Health Network Cancer Institute, 320 E. North Ave, Pittsburgh, PA 15212, USA \\ ${ }^{2}$ Department of Colorectal Surgery, Allegheny Health Network, 320 E. North Ave, Pittsburgh, PA 15212, USA \\ *Author for correspondence: Tel.: +1 412359 3400; Fax: +1 412359 3171; Rodney.wegner@ahn.org
}

\begin{abstract}
Aim: Stereotactic body radiotherapy (SBRT) has been used as an alternative to surgical intervention to treat primary malignanices of the lung as well as lesions from other primaries. In this study, we evaluate the safety and efficacy of SBRT in treating lung metastases from colorectal cancer (CRC). Materials \& methods: We reviewed 22 patients that underwent lung SBRT for metastases from CRC. Almost all patients received chemotherapy before and after undergoing SBRT. Outcomes that were analyzed included overall survival, distant failure and progression-free survival, as well as the effects of biologically effective dose (BED) and KRAS status on local control. Results: Seven females and 15 males underwent SBRT to lung metastases from CRC. The median Eastern Cooperative Oncology Group status was one (0-2). The median dose was 48 Gy (40-54 Gy) in $5 \mathrm{fx}(4-8 \mathrm{fx})$ and the median number of nodules treated with SBRT was one (1-3). Median follow-up was 28.5 months from SBRT and 79 months (9-145) from primary diagnosis. Local control at 1 and 3 years was 75 and $58 \%$, respectively. There was a trend toward improved local control with increasing biologically effective dose $\left(\mathrm{BED}_{10}>100 ; \mathrm{p}=0.07\right)$. Cancers that were positive for the KRAS mutation had increased local control at 12 months, 100 versus $75 \%(p=0.0199)$. Median OS from the primary diagnosis of CRC and from SBRT was 79 and 31 months, respectively. There were no predictors for OS. There were no episodes of acute or late grade 3 or higher toxicity. Conclusion: The results of this study add to the growing body of literature to support SBRT for lung metastases, specifically those patients with limited lung metastases from CRC. The choice of radiation dose remains important, even in metastatic disease, as highlighted by the trend toward improved local control with increasing BED ${ }_{10}$.
\end{abstract}

First draft submitted: 14 March 2018; Accepted for publication: 14 May 2018; Published online: 19 November 2018

Keywords: colorectal cancer $\bullet$ lung metastases $\bullet$ stereotactic body radiotherapy

Colorectal cancer (CRC) remains a common malignancy in the USA, with 140,000 new cases each year and a corresponding 50,000 deaths. Approximately $20 \%$ of patients will have metastatic disease at presentation [1]. It is well established that patients with a limited number of metastases in the liver can experience long-term survival with surgical management, with 5-year survival rates in the 30-50\% range [2]. In addition, studies have shown that metastasectomy for limited lung metastases from CRC can lead to similar rates of disease-free and overall survival (OS) compared with patients with hepatic metastases [3,4].

In medically inoperable, frail patients with primary lung cancers that cannot undergo surgical resection, lung stereotactic body radiotherapy (SBRT) has emerged as a very safe and effective means of providing an ablative therapy with durable local control [5,6]. The excellent results seen in proven and presumed non-small-cell lung cancer (NSCLC) were eventually extrapolated to patients with limited metastatic disease from other primary malignancies. Several series have examined outcomes in such patients managed with SBRT for lung lesions from many types of primary tumors, including CRC. Results of those studies indicate comparable outcomes with primary NSCLC, with low toxicity rates and local control in the $75-90 \%$ range [7-11]. Herein, the authors present outcomes in a series of patients with lung metastases from CRC treated with SBRT. 
Table 1. Patient characteristics.

\begin{tabular}{|c|c|}
\hline Characteristic & Number ( $\%$ or range) \\
\hline Male & $15(68)$ \\
\hline Female & $7(32)$ \\
\hline Median age & $66.5(51-84)$ \\
\hline ECOG & $1(0-2)$ \\
\hline $\begin{array}{l}\text { Oligometastatic } \\
\text { - Yes } \\
\text { - No }\end{array}$ & $\begin{array}{l}18(82) \\
4(18)\end{array}$ \\
\hline $\begin{array}{l}\text { Extrathoracic disease } \\
- \text { Yes } \\
\text { - No }\end{array}$ & $\begin{array}{l}14(64) \\
8(36)\end{array}$ \\
\hline $\begin{array}{l}\text { KRAS status } \\
\text { - Wildtype } \\
\text { - Mutated } \\
\text { - Not documented }\end{array}$ & $\begin{array}{l}5(23) \\
4(18) \\
13(59)\end{array}$ \\
\hline Number of nodules & $1(1-3)$ \\
\hline SBRT dose & 48 Gy (40-54 Gy) \\
\hline $\begin{array}{l}\text { SBRT fractions } \\
\text { BED }_{10}\end{array}$ & $\begin{array}{l}5 \text { (4-8) } \\
100 \text { Gy (60-105.6 Gy) }\end{array}$ \\
\hline Median PTV volume & $14 c c(3-62 c c)$ \\
\hline
\end{tabular}

\section{Materials \& methods}

We identified 22 patients with a total of 27 lesions treated with lung SBRT for lung metastases from CRC between January 2008 and June 2017 in this Institutional Review Board (IRB)-approved retrospective study. A total of 17 patients (77\%) were treated without biopsy based on high clinical suspicion, while five patients (23\%) were treated with tissue confirmation of metastatic disease. Patient characteristics are outlined in Table 1. Patients were typically selected for SBRT based on oligometastatic disease (defined as five or fewer sites of metastasis), with good performance status, that had excellent response to chemotherapy.

The SBRT was delivered in the outpatient setting using dose and fractionation schemes left up to the discretion of the treating radiation oncologist. All patients underwent a $4 \mathrm{D}$ noncontrast chest CT with $1.5-3 \mathrm{~mm}$ slices for treatment planning-simulation to account for respiratory motion. A gross tumor volume was delineated on a free breathing scan and expanded on four expiratory and four inspiratory phases to generate an internal target volume. The planning target volume (PTV) expansion was typically $5 \mathrm{~mm}$, occasionally less if adjacent to the ribs or a central structure, at the discretion of the treating physician. The PTV represents the gross target with margin included for potential setup error and motion, and typically correlates with tumor size. Linear accelerator-based radiotherapy was delivered via 8-12 coplanar 3D conformal beams with $6 \mathrm{MV}$ photons. The median dose for both cohorts was $48 \mathrm{~Gy}$ in four fractions, ranging from 40 to $54 \mathrm{~Gy}$ in four to eight fractions, corresponding to a biologically effective dose using an $\alpha / \beta$ of $10\left(\mathrm{BED}_{10}\right)$ of 60 to $105.6 \mathrm{~Gy}$. The median dose covering $95 \%$ of the PTV was consistent with the prescribed dose. Daily megavoltage cone beam CT was used for image guidance. Figure 1 shows a representative treatment plan.

After treatment, patients were typically followed with noncontrast chest CTs or PET/CTs at least every 3-6 months for 1 year and every 6 months thereafter. Response to treatment and local/distant control was assessed via RECIST criteria. Patient characteristics and size, location and growth were reported if available and correlated with disease progression with univariate and multivariate analysis via Cox regression models [12]. Survival, local control, distant control and freedom from progression were all determined via Kaplan-Meier methodology [13]. All statistics were conducted via MedCalc Version 18.0.

\section{Results}

A total of 15 males and seven females with 27 treated lung lesions from CRC were included in this study. The median age was 66.5 years (range: 51-84) with a median Eastern Cooperative Oncology Group performance status of 1 (range: $0-2$ ). Five patients had pathological confirmation of lung lesions confirming metastatic disease. Nine patients (40\%) had documentation of KRAS status, with five being wildtype and four mutated. The median 
Figure 1. Axial image from treatment plan for stereotactic body radiotherapy to $\mathbf{4 8} \mathrm{Gy}$ in four fractions for lung metastasis from colorectal cancer.

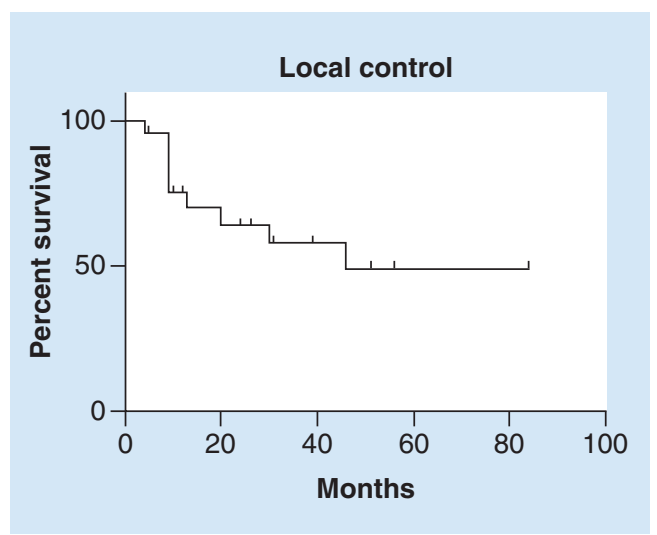

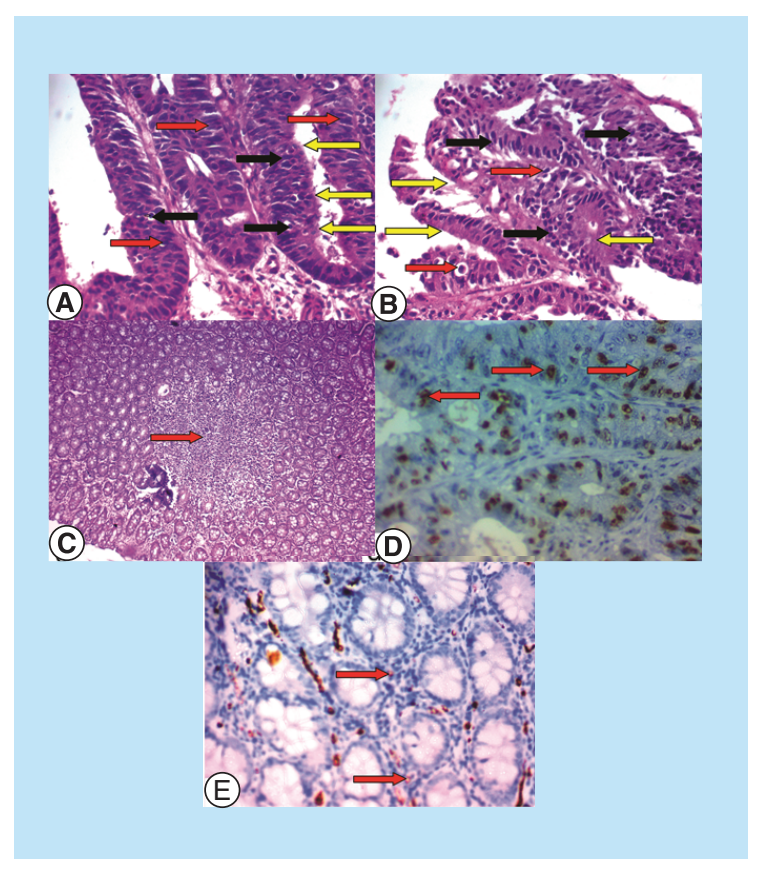

Figure 2. Local control after lung stereotactic body radiotherapy. Median local control was 46 months, with 1-year local control of $75 \%$.

time from diagnosis of CRC to lung metastases was 15 months (range: 0-90). The median time to lung SBRT from primary diagnosis was 33 months (range: 4-117). A total of 14 patients had extrathoracic disease at the time of SBRT, and 18 patients had oligometastatic disease (defined as five or fewer sites of metastasis). Almost all patients (21 of 22) had chemotherapy (typically 5-flourouracil-based) prior to lung SBRT, and 19 of 22 went on to chemotherapy after SBRT. The median number of nodules was 1 (range: 1-3). As above, the median SBRT dose for all patients was $48 \mathrm{~Gy}$ in four fractions, ranging from 40 to $54 \mathrm{~Gy}$ in four to eight fractions, corresponding to a biologically equivalent dose $\left(\mathrm{BED}_{10}\right)$ of 60 to $105.6 \mathrm{~Gy}$. The median PTV volume was $14 \mathrm{cc}$ (range: 2-62 cc).

Median follow-up from SBRT was 28.5 months (range: 1-84) and median follow-up from primary diagnosis was 79 months (range: 9-145 months). Almost all patients (21 of 22) had follow-up chest imaging available for review, with the median number of follow-up scans being six (range: 1-14). Median local control was 46 months with 1-year local control of 75\% and 2-year local control of 65\% (Figure 2). Local control was not influenced by PTV volume (also a surrogate for tumor size), but $\mathrm{BED}_{10} \geq 100$ Gy did show a trend toward improvement in control $(\mathrm{p}=0.07)$. Local control at 1 year was 87 versus $50 \%$ in favor of BED $\geq 100$ Gy (Figure 3). Local control was positively impacted by KRAS mutated status in the nine patients with that information available, with 12-month rates of local control of 100 and $75 \%$, respectively $(\mathrm{p}=0.0199$; not shown). Local control was not affected by PTV volume in this group of patients.

The median OS from SBRT was 31 months for all patients in this study, with 1- and 5-year survivals of 71 and $37 \%$, respectively (Figure 4). There was no difference in OS based on oligometastatic disease or presence of 

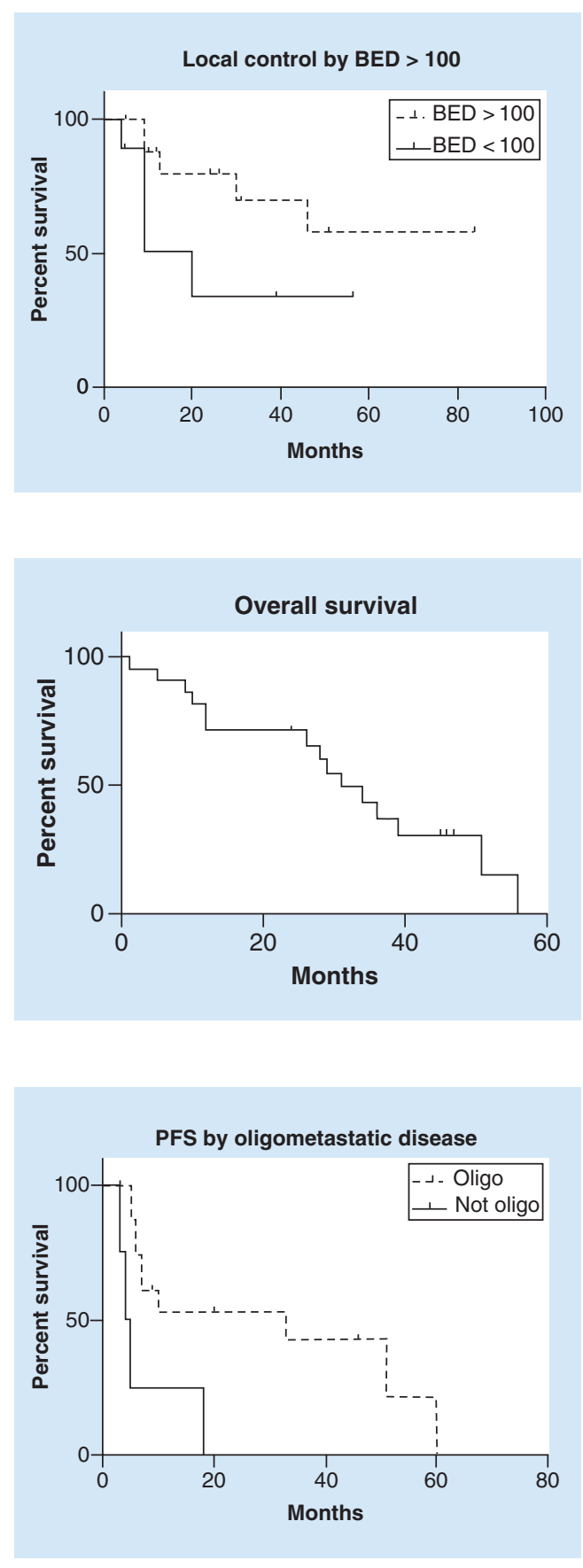

Figure 3. Local control by $\mathrm{BED}_{10} \geq 100 \mathrm{~Gy}$. 1-year local control was 87 versus $50 \%$ in favor of increased $B D_{10} ; p=0.07$. $B E D_{10}$ : Biologically effective dose.

Figure 4. Overall survival from time of stereotactic body radiotherapy. Median survival was 31 months, with 1- and 3 -year rates of 71 and $37 \%$, respectively.

Figure 5. Progression-free survival by oligometastatic disease. The 6-month and 12-month progression-free survival were 75 versus $25 \%$ and 54 versus $25 \%$, respectively, in favor of oligometastatic disease $(p=0.0063)$.

PFS: Progression-free survival.

extrathoracic disease. Similarly, KRAS status did not affect OS in this patient group. The median OS from primary diagnosis was 79 months, with a 5 -year survival rate of $63 \%$. The rate of distant failure was $55 \%$ at 1 year and $68 \%$ at 2 years. Distant control was improved if patients had no extrathoracic disease or had oligometastatic disease. In terms of progression-free survival, the presence of oligometastatic disease significantly predicted improved outcome, with median progression-free survival of 33 months compared with 4.5 months (Figure 5; $\mathrm{p}=0.0063$ ). There were no episodes of acute or late grade 3 or higher toxicity within this cohort throughout the follow-up period.

\section{Discussion}

The CRC remains the third most common malignancy in the USA, with close to 150,000 new cases per year and 50,000 deaths [1]. Patients can present with metastatic disease up to $20-25 \%$ of time, and, as expected, patients with earlier stage disease can progress to metastatic disease throughout the course of their treatment and follow-up. 
Oftentimes, the liver is the dominant site of metastatic disease for CRC. If the number of lesions in the liver are limited, these patients can be treated aggressively and quite successfully with local therapy such as surgery, radiofrequency ablation (RFA) or SBRT [2,14,15].

To date, surgery still provides the highest likelihood of a true 'cure' in patients with limited liver lesions. One surgical series examining outcomes of 100 patients treated in the late 1990s with resection for limited lesions showed a 5 -year OS of $58 \%$, with a $1 \%$ perioperative mortality rate $[2,16]$. The RFA is also a potential option for patients with potentially resectable liver metastases typically measuring less than $3 \mathrm{~cm}$, with local control rates in the $90-98 \%$ range depending on size and long-term survival rates in the $30 \%$ range at 5 years [14,17]. In addition to surgery and RFA, SBRT has emerged as a viable and less invasive ablative technique for limited liver lesions from a separate primary. Multiple series have been published with local control in the $90-95 \%$ range and OS of $60 \%$ at 2 years $[15,18]$.

In addition to the liver, CRC can spread to the lung in the metastatic setting. Given the excellent results seen with an aggressive approach to limited liver metastases, a similar approach has been adopted in limited lung metastases from CRC. As is the case with colorectal liver metastases, surgery remains a viable and effective treatment option. Multiple surgical series have shown outcomes akin to patients with limited hepatic disease burden; with 5-year OS rates around $50 \%$ and high rates of local control $[4,19,20]$. If the patient is older, or has other comorbid conditions, lung surgery may not be feasible. The SBRT initially emerged as a viable alternative for medically inoperable NSCLC, with high rates of local control and minimal toxicity $[5,6]$. That experience, combined with the reasonable success of pulmonary metastatectomy, was then extrapolated to patients with limited lung metastases unable to undergo surgical intervention.

A Phase I/II trial from the University of Colorado enrolled 38 patients with one to three lung metastases from any primary and used three-fraction SBRT, escalating the dose from 48 to 60 Gy [21]. With a median followup of 15 months local control at 1 and 2 years was 100 and $96 \%$, respectively. Toxicity was minimal with a single episode of symptomatic pneumonitis. The group from Rochester also has significant experience treating oligometastatic disease with SBRT [22]. They described results from the combination of two pilot studies for all sites of oligometastasis using varying dose schemes based on anatomic location. The 2-year local control rate overall was $77 \%$. Interestingly, lesions from CRC had poorer local control overall (keeping in mind this study was not limited to lung lesions).

There are a handful of studies looking specifically at lung metastases from CRC, a few of which will be discussed below. The same group from Rochester did report outcomes on SBRT for lung metastases only from CRC [10]. Outcomes from 65 patients treated over a 10-year period were reported. In that study, almost all patients (98\%) developed distant failure and extrathoracic disease independently predicted OS. Median local failure free survival was 15 months in this group of patients, with actuarial local control not reported. An Italian study was published in 2017 reporting outcomes in 44 patients with lung metastases from CRC treated with SBRT, 75\% of which had oligometastatic disease [7]. At 1-year local control was $68 \%$ and at 3 years OS was $50.8 \%$. There was no grade 3 or higher toxicity. In this study, multiple lung nodules and metastasis at presentation were associated with worse outcomes. And finally, another group from Italy also performed a comparative cohort study in a retrospective fashion comparing outcomes between patients with lung metastases from CRC treated either surgically or with SBRT [9]. The study had 142 patients treated with surgery and only 28 managed with SBRT. As expected, the patients in the SBRT cohort were older. After 2 years, there was no significant difference in OS, 77 (SBRT) versus $82 \%$ (surgery; $\mathrm{p}=0.134$ ). Local control was not reported in that study. The authors concluded that OS appeared to be similar, at least with 2 years of follow-up, for patients managed with SBRT in lieu of surgery.

The results presented in this study mirror those discussed above. Patients treated at our institution with lung SBRT for limited lung metastases experienced excellent local control of $75 \%$ at 1 year with no severe acute or late toxicity. Similar to NSCLC, we did show a trend toward improved local control $(p=0.07)$ for dosing schemes with a $\mathrm{BED}_{10}>100$, highlighting the importance of dose selection, even in the metastatic setting. Local control in our series also appeared to be affected by KRAS status, with patients harboring the mutation experiencing better local control. That result must be interpreted with caution, however, as our sample number was small (nine patients total with status documented) and could lead to overestimation of its effect. A total of 5-year OS in our study from time of SBRT was on par with other oligometastatic series, with a rate of $37 \%$. Given the nature of metastatic disease, not surprisingly $55 \%$ of patients experienced distant failure at 1 year, highlighting the importance of continued close surveillance after treatment. As expected, patients with true oligometastatic disease had lower rates of any progression, $45 \%$ compared with $75 \%$ at 1 year. That difference in outcome highlights the importance of 
patient selection, and that SBRT should be selectively offered to those patients with 1-5 sites of metastatic disease. The limitations of our study are those inherent to retrospective series including selection bias. In addition, when dealing with patients with metastatic disease, distant failure and death from nonpulmonary causes are significant competing factors, which can perhaps skew local control results. This factor must be taken into consideration when considering results of studies done in this type of patient population.

\section{Conclusion}

Lung SBRT remains a viable treatment option for patients with limited metastatic disease from CRC, with high rates of local control and minimal toxicity. This study highlights the fact that dose selection and patient selection are important, favoring effective $\mathrm{BED}_{10} \mathrm{~s}$ and patients with true oligometastatic disease. Properly made decisions in regard to those factors will lead to the best outcomes.

\section{Summary points}

- Stereotactic body radiotherapy (SBRT) is a noninvasive technique to deliver ablative doses of radiation.

- The SBRT was initially utilized in early stage non-small-cell lung cancer but now represents a viable option for limited lung metastases.

- This study presents outcomes in 22 patients with limited lung metastases from colorectal cancer.

- The median dose was 48 Gy delivered over four to five fractions with a median follow-up of 28.5 months.

- A 1-year local control was excellent at $75 \%$.

- There was a trend toward improved local control with increased biologically effective dose.

- Median overall survival from SBRT was 31 months and there was no significant acute or late toxicity.

- The SBRT remains a viable treatment option for patients with limited lung metastases from colorectal cancer.

Financial \& competing interests disclosure

The authors have no relevant affiliations or financial involvement with any organization or entity with a financial interest in or financial conflict with the subject matter or materials discussed in the manuscript. This includes employment, consultancies, honoraria, stock ownership or options, expert testimony, grants or patents received or pending, or royalties.

No writing assistance was utilized in the production of this manuscript.

Ethical conduct of research

The authors state that they have obtained appropriate institutional review board approval or have followed the principles outlined in the Declaration of Helsinki for all human or animal experimental investigations.

\section{Open access}

This work is licensed under the Attribution-NonCommercial-NoDerivatives 4.0 Unported License. To view a copy of this license, visit http://creativecommons.org/licenses/by-nc-nd/4.0/

\section{References}

1. Siegel RL, Miller KD, Jemal A. Cancer statistics, 2018. CA Cancer J. Clin. 68(1), 7-30 (2018).

2. Fernandez FG, Drebin JA, Linehan DC, Dehdashti F, Siegel BA, Strasberg SM. Five-year survival after resection of hepatic metastases from colorectal cancer in patients screened by positron emission tomography with F-18 fluorodeoxyglucose (FDG-PET). Ann. Surg. 240(3), 438-447; discussion 47-50 (2004).

3. Okumura T, Boku N, Hishida $\mathrm{T}$ et al. Surgical outcome and prognostic stratification for pulmonary metastasis from colorectal cancer. Ann. Thorac. Surg. 104(3), 979-987 (2017).

4. Kim HK, Cho JH, Lee HY, Lee J, Kim J. Pulmonary metastasectomy for colorectal cancer: how many nodules, how many times? World J. Gastroenterol. 20(20), 6133-6145 (2014).

5. Baumann P, Nyman J, Hoyer M et al. Outcome in a prospective phase II trial of medically inoperable stage I non-small-cell lung cancer patients treated with stereotactic body radiotherapy. J. Clin. Oncol. 27(20), 3290-3296 (2009).

6. Timmerman R, Paulus R, Galvin J et al. Stereotactic body radiation therapy for inoperable early stage lung cancer. JAMA 303(11), 1070-1076 (2010). 
7. Agolli L, Bracci S, Nicosia L, Valeriani M, De Sanctis V, Osti MF. Lung metastases treated with stereotactic ablative radiation therapy in oligometastatic colorectal cancer patients: outcomes and prognostic factors after long-term follow-up. Clin. Colorectal Cancer 16(1), 58-64 (2017).

8. Carvajal C, Navarro-Martin A, Cacicedo J, Ramos R, Guedea F. Stereotactic body radiotherapy for colorectal lung oligometastases: preliminary single-institution results. J. BUON 20(1), 158-165 (2015).

9. Filippi AR, Guerrera F, Badellino $S$ et al. Exploratory analysis on overall survival after either surgery or stereotactic radiotherapy for lung oligometastases from colorectal cancer. Clin. Oncol. (R. Coll. Radiol.) 28(8), 505-512 (2016).

10. Qiu H, Katz AW, Chowdhry AK et al. Stereotactic body radiotherapy for lung metastases from colorectal cancer: prognostic factors for disease control and survival. Am. J. Clin. Oncol. 41(1), 53-58 (2018).

11. Rieber J, Streblow J, Uhlmann L et al. Stereotactic body radiotherapy (SBRT) for medically inoperable lung metastases - A pooled analysis of the German working group "stereotactic radiotherapy". Lung Cancer 97, 51-58 (2016).

12. Cox DR. Regression models and life- tables. J. Roy. Statist. Society 34(2), 87-220 (1972).

13. Kaplan EL, Meier P. Nonparametric estimation from incomplete observations. J. Am. Stat. Assoc. 53(282), 457-481 (1958).

14. Gillams AR, Lees WR. Radio-frequency ablation of colorectal liver metastases in 167 patients. Eur. Radiol. 14(12), 2261-2267 (2004).

15. Kirichenko A, Gayou O, Parda D et al. Stereotactic body radiotherapy (SBRT) with or without surgery for primary and metastatic liver tumors. HPB (Oxford) 18(1), 88-97 (2016).

16. Wei AC, Greig PD, Grant D, Taylor B, Langer B, Gallinger S. Survival after hepatic resection for colorectal metastases: a 10-year experience. Ann. Surg. Oncol. 13(5), 668-676 (2006).

17. Abitabile P, Hartl U, Lange J, Maurer CA. Radiofrequency ablation permits an effective treatment for colorectal liver metastasis. Eur. J. Surg. Oncol. 33(1), 67-71 (2007).

18. Caivano D, Valeriani M, Russo I et al. Stereotactic body radiation therapy in primary and metastatic liver disease. Anticancer Res. 37(12), 7005-7010 (2017).

19. Wiegering A, Riegel J, Wagner J et al. The impact of pulmonary metastasectomy in patients with previously resected colorectal cancer liver metastases. PLoS ONE 12(3), e0173933 (2017).

20. Zabaleta J, Aguinagalde B, Lopez I, Fernandez-Monge A, Izquierdo JM, Emparanza JI. Survival after pulmonary metastasectomy in colorectal cancer patients: does a history of resected liver metastases worsen the prognosis? A literature review. Cancer Biol. Med. 14(3), 281-286 (2017).

21. Rusthoven KE, Kavanagh BD, Burri SH et al. Multi-institutional Phase I/II trial of stereotactic body radiation therapy for lung metastases. J. Clin. Oncol. 27(10), 1579-1584 (2009).

22. Milano MT, Katz AW, Schell MC, Philip A, Okunieff P. Descriptive analysis of oligometastatic lesions treated with curative-intent stereotactic body radiotherapy. Int. J. Radiat. Oncol. Biol. Phys. 72(5), 1516-1522 (2008). 
